\title{
MEDICAL IMAGE ANALYSIS AND PROCESSING USING A DUAL TRANSFORM
}

\author{
Shiby Angel K ${ }^{1}$ \\ ${ }^{1}$ Assistant Professor, Karunya University, Coimbatore
}

\begin{abstract}
The demand for images in medical field has increased drastically over the years. The need for reducing the storage space has resulted in image compression. This paper presents a dual transform for medical image compression algorithm. The experimental results determines how the compression ratio (CR), peak signal to noise ratio (PSNR) and SNR (signal to noise ratio) of different compression algorithms responds to dual transform algorithm.
\end{abstract}

Keywords: DCT, SPIHT, Haar Wavelet, Linear approximation transform, image compression, Singular Value Decomposition (SVD).

\section{INTRODUCTION}

Image compression, an important area in the field of digital image processing deals with techniques for reducing the storage required for saving an image or the bandwidth required for transmitting. The aim of image compression is to reduce the number of bits required to represent an image and is done by removing the redundancies. The degree of redundancy determines how much compression can be achieved.

The main classification of compression includes lossy and lossless compression. Lossy compression compresses data by discarding some of the data while lossless compression does not change the content of the file. Lossless compression is mainly preferred for archival purposes and medical imaging. Medical images are of interest for a large number of applications and it resulted in a constant growth in the amounts of data that have to be transmitted, processed, and stored efficiently [1].

The people in India, particularly in rural and remote areas are found struggling to access timely medical treatment. The region of the country is characterized by densely populated communities spread over vast distances; there is a lack of qualified personnel in certain sectors of the health service.

Telemedicine has come originally to serve rural populations, or any people who are geographically dispersed, where time and the cost of travel make access to the best medical care difficult. In this paper an efficient method for compression of medical images is compared and studied with different metrics and algorithms.

\section{BACKGROUNDS}

SVD is one of the most useful tools of linear algebra [2] compared to other linear approximation methods. SVD is a factorization and approximation technique which effectively reduces any matrix into a smaller invertible and square matrix. The SVD matrix decomposition which is extensively used in mathematics [3] appears in fields related directly with algebra, such as least squares problems or the calculus of the matrix rank. Its usefulness in applications concerning image processing includes pattern recognition, secret communication of digital images, movement estimation, quantization, and compression of images and video sequences.

SVD is commonly used in face recognition, object detection, field matching techniques and meteorological and oceanographic data analysis. One of the main features that made SVD to have application in image processing is that it is a factorization and approximation technique which effectively reduces any matrix into a smaller invertible and square matrix [3]. SVD was also used in the area of face recognition which proved some properties that the SVD approach is robust, simple, easy and fast to implement [5]. SVD when compared with wavelet compression, block truncating compression, and discrete cosine transform compression provided evidence that when singular value increases, the size and quality of compressed image improves [6]. Similarly the performance analysis of SVD Algorithm portrayed that using SVD computation, an image matrix can be compressed to a significantly smaller sized matrix and during decomposition the image is almost identical to original image. This in turn saves a lot of memory space [7]. 
Haar transform, a simplest compression process of wavelet, is a multi scale transformation [12]. Wavelets are functions generated from a single function by its dilations and translations [13]. Haar wavelet has special properties such as robust, high compression ratios and provides good results in terms of imperceptibility which makes it use mainly in watermarking [14]. DCT transform introduces no loss to the source image samples; it transforms them to a domain in which they can be more efficiently encoded.

There are several encoding methods that provide high image quality at high compression rates. The SPIHT image coding algorithm developed in 1996 [15] is another more efficient implementation of the embedded zero tree wavelet (EZW) algorithm [16]. SPIHT provides even better performance [17] than the other extensions of EZW. EBCOT [18] which is a block based encoder is more complicated and also timeconsuming [24]. SPECK [19] also which uses recursive setpartitioning procedure to sort subsets of wavelet coefficients by maximum magnitude with respect to integer powers of two thresholds. When compared to other encoders mentioned above SPIHT provides salient features such as better quality, visually superior, intensive progressive capability, SNR scalability and, low computational complexity [20,21,22,23]. The computational speed of the SPIHT algorithm is also found to be very good [23].

\section{DUAL TRANSFORM}

\subsection{Singular Value Decomposition \& Haar-Wavelet}

\section{Transform}

SVD, extensively used in mathematics is a factorization of a real or complex matrix in linear algebra. One of the special features of SVD is that it can be performed on any real $\mathrm{m} \times \mathrm{n}$ matrix. It factorizes matrix $\mathrm{A}$ into three matrices $\mathrm{U}, \mathrm{S}$ and $\mathrm{V}$ where, $\mathrm{U}$ is a left singular matrix and $\mathrm{V}$ is the right singular matrix and $\mathrm{S}$ is a diagonal matrix. $\mathrm{U}, \mathrm{S}$ and $\mathrm{V}$ are obtained by calculating rank, eigen values and eigen vectors of the matrix A. The process of SVD begins by selecting the matrix $A(m x$ $\mathrm{n})$ which has $\mathrm{m}$ rows and $\mathrm{n}$ columns. Now, matrix $\mathrm{A}$ is factorized into three matrices $\mathrm{U}, \mathrm{S}$ and $\mathrm{V}^{\mathrm{T}}$.

$$
\mathrm{A}=\mathrm{USV}^{\mathrm{T}}
$$

Wavelet transform or wavelet analysis is a very efficient approach developed as a mathematical tool for signal analysis. The Haar Transform technique widely used in wavelet analysis is the simplest wavelet. Haar wavelet transform has been used as an earliest example for orthonormal wavelet transform with compact support. The haar transform (HT) is one of the simplest and basic transformations from a space domain are a local frequency domain and it reduces the calculation work [27]. HT decomposes the linear approximated image as approximation components and detail components.

\subsection{Encoding Process}

Once the linear approximated image is transformed using haar wavelet, the coefficients are further decomposed using the SPIHT encoder. Salient features of SPIHT makes it visually superior and of better quality. After the haar wavelet transform is applied to an image, the SPIHT algorithm works by partitioning the wavelet decomposed image into significant and insignificant partitions based on the following function:

$$
s_{n}(U)=\left\{\begin{array}{l}
1, \max \left|C_{i, j}\right| \geq 2^{n} \\
0, \text { otherwise }
\end{array}\right.
$$

Where $s_{n}(U)$ is the significance of a set of co-ordinates $\mathrm{T}$, and $c_{i, j}$ is the coefficient value at co-ordinate $(i, j)$. The block diagram of dual transform and SPIHT encoder is seen in Fig.1.

\section{Algorithm: Dual Transform based Image Compression}

Step1: Data-Input image $\mathrm{A}(\mathrm{m}, \mathrm{n})$ of size $256 \times 256$ which is a matrix $\mathrm{A}(\mathrm{m} \times \mathrm{n})$

Step2: Apply dual transform:

a) Compute its transpose $\mathrm{A}^{\mathrm{T}}$ and $\mathrm{A}^{\mathrm{T}} \mathrm{A}$.

b) Determine the eigen values of $\mathrm{A}^{\mathrm{T}} \mathrm{A}$ and sort these in descending order, in the absolute sense. Square roots these to obtain the singular values of $\mathrm{A}$.

c) Construct diagonal matrix $\mathrm{S}$ by placing singular values in descending order along its diagonal. Compute its inverse, $\mathrm{S}^{-1}$.

d) Use the ordered eigen values from step $b$ and compute the eigenvectors of $\mathrm{A}^{\mathrm{T}} \mathrm{A}$. Place these eigenvectors along the columns of $\mathrm{V}$ and compute its transpose, $\mathrm{V}^{\mathrm{T}}$.

e) Compute $U$ as $U=A V S^{-1}$. Now, compute the full SVD using $\mathrm{A}=\mathrm{USV}^{\mathrm{T}}$.

f) Decompose the linearly approximated image using haar wavelet transform.

Step 3: Encode the resultant coefficients using SPIHT encoder.

Step 4:Calculate the resulting image quality of the compressed images using different metrics such as Peak Signal to Noise Ratio (PSNR), Mean Square Error (MSE), Bits Per Pixel (BPP), Compression ratio (CR) and Computational time (CT).

\section{PERFORMANCE EVALUATION}

To evaluate the performance of dual transform with other transforms, three medical images of size $256 \times 256$ with 8 bits per pixel are used. The efficiency is compared with Haar Wavelet-SPIHT [28] and DCT-SPIHT [29] for different metrics such as PSNR, MSE, CR, CT and BPP. Table 1-3 shows the performance of different methods using the 3 
medical images. Fig. 4-6 shows the rate vs. PSNR performance of the 3 different medical images. Bit rate is the average bits required to represent a single pixel in the compressed image. It's obviously clear from the graphs that the PSNR value increases with increase in bits per pixel.

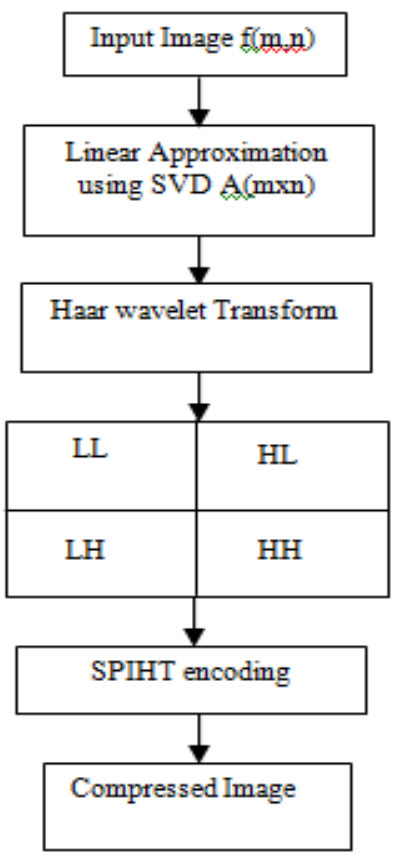

Fig-1: The block diagram of the proposed compression method

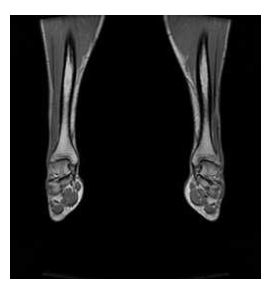

a)

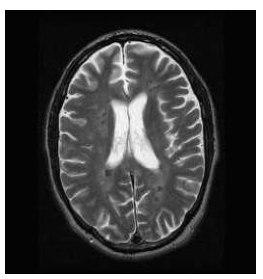

b)

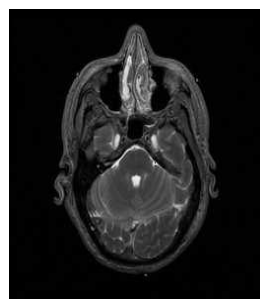

c)

a) T1WIAnkle b) T2WI-Axial-1 c) T2WI-Axial -2

Fig-2: Original Images
Table-1: MSE values attained by dual transform and Existing methods at $0.4 \mathrm{bpp}$

\begin{tabular}{|l|c|c|c|}
\hline $\begin{array}{c}\text { Compressi } \\
\text { on } \\
\text { Methods }\end{array}$ & $\begin{array}{c}\text { T1WIAnkl } \\
\mathbf{e}\end{array}$ & $\begin{array}{c}\text { T2WIAxia } \\
\mathbf{l - 1}\end{array}$ & $\begin{array}{c}\text { T2WI-Axial } \\
\mathbf{- 2}\end{array}$ \\
\hline $\begin{array}{l}\text { Haar- } \\
\text { SPIHT }\end{array}$ & 1.77 & 2.3 & 1.4 \\
\hline $\begin{array}{l}\text { DCT- } \\
\text { SPIHT }\end{array}$ & 1.73 & 2.9 & 3.35 \\
\hline \begin{tabular}{l} 
Proposed \\
\hline
\end{tabular} & 1.43 & 1.95 & 1.08 \\
\hline
\end{tabular}

Table-2: CR values attained by dual transform and Existing methods at $0.4 \mathrm{bpp}$

\begin{tabular}{|c|c|c|c|}
\hline $\begin{array}{c}\text { Compressi } \\
\text { on } \\
\text { Methods }\end{array}$ & $\begin{array}{c}\text { T1WIAn } \\
\text { kle }\end{array}$ & $\begin{array}{c}\text { T2WI- } \\
\text { Axial-1 }\end{array}$ & $\begin{array}{c}\text { T2WI-Axial } \\
\mathbf{- 2}\end{array}$ \\
\hline $\begin{array}{c}\text { Haar- } \\
\text { SPIHT }\end{array}$ & 12.02 & 11.0 & 13.44 \\
\hline $\begin{array}{c}\text { DCT- } \\
\text { SPIHT }\end{array}$ & 8.87 & 7.84 & 10.46 \\
\hline Proposed & 16.92 & 13.75 & 16.15 \\
\hline
\end{tabular}

Table-3: PSNR values attained by dual transform and Existing methods at $0.4 \mathrm{bpp}$

\begin{tabular}{|c|c|c|c|}
\hline $\begin{array}{c}\text { Compressi } \\
\text { on } \\
\text { Methods }\end{array}$ & $\begin{array}{c}\text { T1WIAn } \\
\text { kle }\end{array}$ & $\begin{array}{c}\text { T2WI- } \\
\text { Axial-1 }\end{array}$ & $\begin{array}{c}\text { T2WI-Axial } \\
\mathbf{- 2}\end{array}$ \\
\hline $\begin{array}{c}\text { Haar- } \\
\text { SPIHT }\end{array}$ & 45.65 & 44.51 & 46.68 \\
\hline $\begin{array}{c}\text { DCT- } \\
\text { SPIHT }\end{array}$ & 45.74 & 43.51 & 42.88 \\
\hline Proposed & 46.59 & 45.23 & 47.81 \\
\hline
\end{tabular}

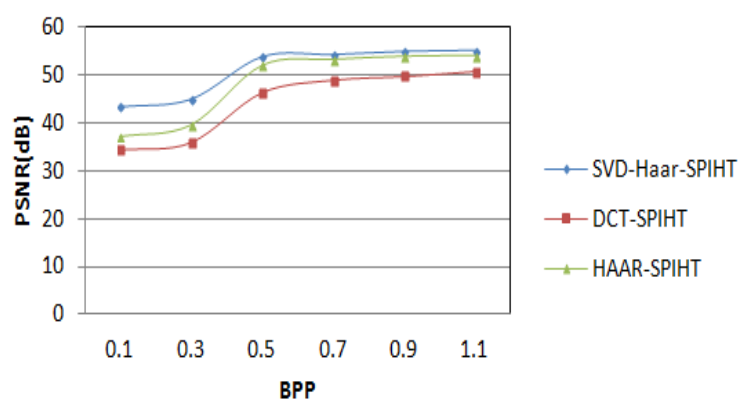

Fig-4: Rate-PSNR performance of T1W1Ankle image obtained using dual transform and existing methods 


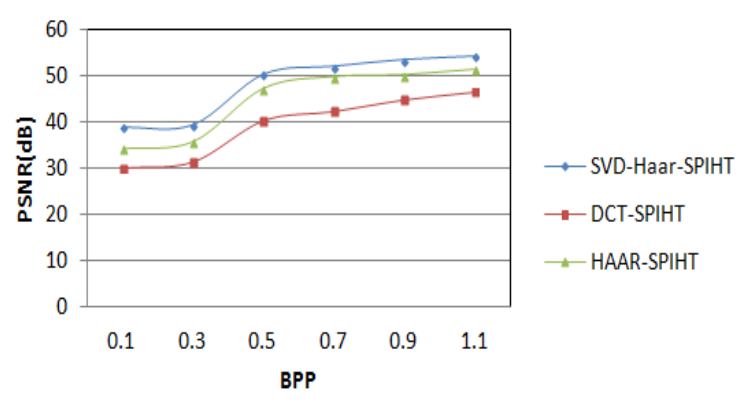

Fig-5: Rate-PSNR performance of T2W1-Axial-1 image obtained using dual transform and existing methods

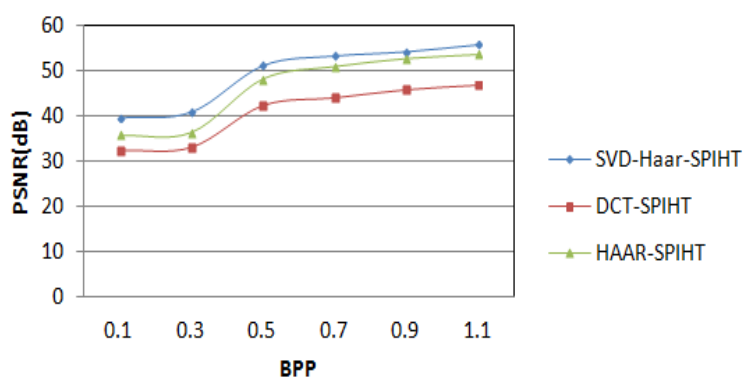

Fig.6 Rate-PSNR performance of T2W1-Axial-2 image obtained using dual transform and existing methods

\section{CONCLUSIONS}

Thus a dual transform for medical image compression was studied. This dual transform method highly preserves quality of images with high compression ratio and bits per pixel. The quality of the image is also improved in terms of PSNR.

In the case of medical images there is a great need to have no deterioration in image quality. The results proved that with no image quality loss, it can be used in the applications of medical purposes. It can also be used to compress images used in telemedicine applications.

\section{REFERENCES}

[1] Xiaojun, John M. Tyler and Oleg S. , "Diagnostically lossless medical image compression via wavelet-based background noise removal", SPIE Proceedings, Vol. 4056, 5 April 2000

[2] Azam sabaghi, Khashayar and Reza Sabbaghi, "Medical Image Watermarking based on SVD-DWT technique", International Congress on Informatics, Environment, Energy and Applications, vol.38, pp.7480,2012

[3] Rajesh A. Jadav and Shailesh S. Patel, "Application of singular value decomposition in image processing", Indian Journal of Science and Technology, Vol. 3 No. 2, pp. 148-150, Feb 2010
[4] Jiani, Jennifer, Xiaofei and Qiang, "Randomized svd methods in hyperspectral imaging", Journal of Electrical and Computer Engineering Volume 2012, pp. 354-379, 2012.

[5] Andrews.H, "Singular Value Decomposition and Digital Image Processing", IEEE Transactions on Acoustics, Speech and Signal Processing, Vol: 24 , Issue: 1 ,pp. $26-53,2003$

[6] Dinesh Gupta,. Pardeep Singh, Nivedita and Sugandha Sharma, "A comparative study of image compression between Singular value decomposition, Block truncating coding, Discrete cosine transform and Wavelet" International Journal of Computer Science and Network Security(IJCSNS), vol.12 No.2, pp.100106, February 2012

[7] Nivedita and Sonika Jindal, "Performance Analysis of SVD and SPIHT Algorithm for Image Compression Application" International Journal of Advanced Research in Computer Science and Software Engineering, Volume 2, issue 2, February 2012

[8] Rehna V.J, Abhranil Dasgupta , "JPEG Image Compression using Singular Value Decomposition", International Journal of Advances in Electronics Engineering, Vol:1 Issue:1 I, pp 81-86,

[9] Tutorial on singular value decomposition and principal component analysis in image processing

[10] Chou-Hao Hsu, "SVD-Based Projection for Face Recognition", IEEE EIT 2007 Proceedings, pp. 600603, 2007

[11] S.Ramakrishnan, T.Gopalakrishnan and K.Balasamy, "A Wavelet Based Hybrid SVD Algorithm for Digital Image Watermarking, Signal \& Image Processing”, An International Journal (SIPIJ), Vol.2, No.3, pp 157-174, September 2011

[12] Chih-Chin La "An improved SVD-based watermarking scheme using human visual characteristics", Optics Communications, Vol. 284, Issue 4, pp. 938-944, 15 February 2011

[13] Andrea , Ales Prochazka and Martina , Article on "Wavelet Based Image Segmentation"

[14] Anuradha, Rudresh Pratap Singh, "DWT Based Watermarking Algorithm using Haar Wavelet", International Journal of Electronics and Computer Science Engineering, Vol.1,No. 1

[15] Said and W. Pearlman, "A new fast and efficient image codec based on set partitioning in hierarchical trees", IEEE Trans. Circuits Syst. Video Technol, vol. 6, pp. 243-250, June 1996

[16] J.M. Shapiro, "Embedded image coding using zerotrees of wavelet coefficients," IEEE Trans. Signal Processing, vol. 41, pp. 3445-3463, Dec.1993

[17] Xaoli Tang, William A. Pearlman and James W. Modestino, A draft on "Hyperspectral Image Compression Using Three-Dimensional Wavelet Coding", March 2004 
[18] D. Taubman, "High performance scalable image compression with EBCOT", IEEE Trans. on Image Processing, vol. 9, No. 7, pp.1158-70, Jun 2000

[19] W.A. Pearlman, A. Islam, N. Nagaraj and A.Said, "Efficient, low complexity image coding with a setpartitioning embedded block coder", IEEE Trans. Circuits and Syst. Video Technol., Vol 14, No 11, pp. 1219 - 1235, Nov. 2004

[20] Bibhuprasad Mohanty, Abhishek Singh, Sudipta Mahapatra, "A High Performance Modified SPIHT for Scalable Image Compression”, International Journal of Image processing (IJIP), Vol (5) : Issue (4), pp. 390402, 2011

[21] Vasuki, P.T. Vanathi, "Progressive Image Compression Using Contourlet Transform ", International Journal of Recent Trends in Engineering, Vol 2, No. 5,pp. 193 197 November 2009.

[22] Veenadevi.S.V. and A.G.Ananth , "Fractal Image Compression Using Quad Tree Decomposition and Huffman Coding", Signal \& Image Processing: An International Journal (Sipij), Vol.3, No.2, pp. 207-212, April 2012

[23] K. Siva Nagi Reddy, B. Raja Sekher Reddy, G. Rajasekhar and K. Chandra Rao , "A Fast Curvelet Transform Image Compression Algorithm using with Modified SPIHT", International Journal of Computer Science and Telecommunications, Vol 3, Issue 2, pp.18, February 2012

[24] C. J. Lian, K. F. Chen, H. H. Chen, and L. G. Chen, "Analysis and architecture design of block-coding engine for EBCOT in JPEG2000," IEEE Trans. Circuits Syst. Video Technol., vol. 13, no. 3, pp. 219230, Mar. 2003

[25] Wanli Ouyang, W.K. Cham, "Fast Algorithm for Walsh Hadamard Transform on Sliding Windows", IEEE Transactions on Pattern Analysis and Machine Intelligence, vol.32, pp.165-171, Jan 2010

[26] Ziad M. Hafed And Martin D. Levine, "Face Recognition Using the Discrete Cosine Transform", International Journal of Computer Vision, vol. 43(3), pp. 167-188, 2001.

[27] Navjot Kaur and Preeti Singh, "Article: Enhancement of Compression Ratio and Image Quality using ISPIHT with MFHWT", International Journal of Computer Applications, vol. 54(8), pp.20-23, September 2012.

[28] Sanjeev Kumar, Varun Sood, "Quality Assessment of Colour Image Compression using Haar Wavelet Transform", International Journal of Engineering Trends and Technology- Vol.3Issue3, pp. 266-269, 2012

[29] Jing Zhang, Guizhong Liu, "A Novel Classified Residual Dct For Hyperspectral Images Scalable Compression", in the proceedings of PCS2007, Portugal.

[30] N. Ahmed, T. Natarjan, "Discrete Cosine Transforms". IEEE trans. Computers, c-23, 1974, pp. 90-93 